\begin{tabular}{ll|l} 
Case Reports in & \multicolumn{2}{c}{ Case Rep Gastroenterol 2020;14:7-14 } \\
\cline { 2 - 3 } Gastroenterology & $\begin{array}{l}\text { DOl: 10.1159/000505090 } 2020 \text { The Author(s) } \\
\text { Published online: January 20, } 2020\end{array}$ & $\begin{array}{l}\text { Published by S. Karger AG, Basel } \\
\text { www.karger.com/crg }\end{array}$ \\
\cline { 2 - 3 } & $\begin{array}{l}\text { This article is licensed under the Creative Commons Attribution-NonCommercial } 4.0 \\
\text { International License (CC BY-NC) (http://www.karger.com/Services/OpenAccessLicense). } \\
\text { Usage and distribution for commercial purposes requires written permission. }\end{array}$
\end{tabular}

\title{
Rectal Arteriovenous Malformation Treated by Transcatheter Arterial Embolization
}

\author{
Sho Ishikawa ${ }^{a}$ Shoichiro Mukai ${ }^{a}$ Yuzo Hirata ${ }^{a}$ Akihiro Kohata ${ }^{a}$ \\ Azusa Kai ${ }^{\mathrm{a}}$ Yosuke Namba ${ }^{\mathrm{a}}$ Sho Okimoto ${ }^{\mathrm{a}}$ Seiji Fujisaki ${ }^{\mathrm{a}}$ \\ Saburo Fukuda ${ }^{a}$ Mamoru Takahashi $^{a}$ Toshikatsu Fukuda $^{a}$ \\ Hideki Ohdan ${ }^{\mathrm{b}}$ \\ ${ }^{a}$ Department of Surgery, Chugoku Rosai Hospital, Kure, Japan; ${ }^{\text {bDepartment of }}$ \\ Gastroenterological and Transplant Surgery, Graduate School of Biomedical and \\ Health Sciences, Hiroshima University, Hiroshima, Japan
}

\section{Keywords}

Arteriovenous malformation - Transcatheter embolization - Interventional radiology .

Hematochezia

\begin{abstract}
An 86-year-old man who presented with frequent hematochezia with mild anemia on blood tests was admitted to our hospital. Colonoscopy exhibited a submucosal tumor-like lesion in the lower rectum. CT and MRI showed blood flow into the lesion, but not tumor component. Angiography of the superior rectal artery and left internal iliac artery showed vascular hyperplasia and nidus. Thus, rectal arteriovenous malformation was diagnosed. If bleeding from arteriovenous malformation was out of control, surgical resection was necessary. However, due to the age of the patient, we performed transcatheter arterial embolization and abdominoperineal resection was not needed. Embolization from the left superior rectal artery, middle rectal artery and inferior rectal artery was performed to control the bleeding and to avoid surgery. After embolization, he was followed up for 10 months in our hospital without recurrence.
\end{abstract}




\section{Introduction}

Arteriovenous malformation (AVM) is a common disease that causes gastrointestinal bleeding; however, AVM occurring in the rectum is very rare. AVM is a vascular malformation, i.e., a shunt and anastomosis of microvessels do not flow through capillaries between arteries. AVM generally consists of an inflow artery, a collection of abnormal blood vessels, and an outflow vein $[1,2]$. It usually occurs in the colon and small intestine but rarely in the rectum. In general, surgical resection has been performed for the treatment of AVM, but in recent years there have been increasing reports of transcatheter arterial embolization (TAE). If AVM occurs in the lower rectum, surgical resection such as abdominoperineal resection (APR) is often performed. Surgical resection is very invasive and reduces the patient's quality of life (QOL). On the other hand, TAE is less invasive than surgical resection and helps to improve the patient's QOL. Described below is a case report of rectal AVM. By performing TAE, a massively invasive surgery may be avoided and can be an alternative treatment for difficult surgical cases. We reviewed the literature to identify the clinical and radiological features of rectal AVM.

\section{Case Report}

An 86-year-old Japanese man was admitted to our hospital due to frequent hematochezia with mild anemia. He had been diagnosed with bleeding hemorrhoids treated by rubber ligation. He had been followed up at his nearby hospital for several years, but since he suffered more frequent hematochezia, he was admitted to our hospital for evaluation and treatment of hematochezia. He had mild anemia ( $\mathrm{Hb} 11.3 \mathrm{~g} / \mathrm{dL}$ ) and active bleeding during rectal examination. Laboratory data showed mild anemia. Colonoscopy revealed a lesion like a submucosal tumor in the lower rectum. There were no findings suggesting the presence of malignant tumors. The lesion had a size of $20 \mathrm{~mm}$ and was localized just above the dentate line (Fig. 1a, b). Endoscopic ultrasonography revealed that the lesion was located outside of the rectum wall and there was blood flow in the lesion (Fig. 1c, d). Contrast-enhanced computed tomography (CECT) showed an thickened rectal wall and vascular hyperplasia around the rectum (Fig. 2a, b), but no tumor component. On T1WI and T2WI obtained by magnetic resonance imaging (MRI), a low signal area was found in the thickened rectal lesion indicating flow voids (Fig. 2c, d). This finding strongly suggested that it was a vascular lesion, not a tumor. Angiography of the inferior mesenteric artery and the internal iliac artery confirmed vascular hyperplasia and nidus in the periphery of the superior rectal artery (SRA) (Fig. 3, 4). Based on these findings, we diagnosed rectal AVM. Since bleeding from AVM was out of control, surgical resection was necessary. However, due to the age of the patient, we performed TAE although APR would have been necessary for the resection of the lesion. TAE is less invasive than surgical resection and helps to improve the patient's QOL. Therefore, we performed TAE to stop the bleeding. Selective angiography of the left branch of the SRA (Fig. 4a) and left internal iliac artery using a microcatheter showed vascular hyperplasia and nidus in the periphery of those arteries (Fig. $4 a, b)$. Embolization was performed from the left SRA, middle rectal artery (MRA), and inferior rectal artery (IRA) with microspheres (Embosphere ${ }^{\circledR}$ ) diluted with contrast medium. After successful embolization, angiography showed obliteration of the blood flow into the AVM (Fig. $4 \mathrm{c}, \mathrm{d})$. After TAE, he was followed up at our hospital once in 10 months for a year and had no recurrence of hematochezia. 


\section{Discussion}

AVM is a vascular malformation, i.e. a short circuit and anastomosis do not flow through capillaries between arteries. AVM generally consist of an inflow artery (feeder), a collection of abnormal blood vessels (nidus), and an outflow vein (drainage route) [1, 2]. There are many reports of AVM occurring in the digestive tract, and the occurrence site is $56 \%$ in the colon including the rectum and $43 \%$ in the small intestine [3]. Rectal AVM is extremely rare. According to Kobayashi et al. [3], typical symptoms of intestinal AVM are intermittent bloody stools without abdominal pain and anemia. There are some cases requiring blood transfusion [4-6]. In our case, the patient complained of frequent bloody stools and admitted mild anemia at the hospital visit.

Abdominal angiography is useful for the diagnosis. The findings are as follows: (1) abnormal dense staining of the intestinal wall in the arterial phase, (2) early thickening of the drainage vein, and (3) angiography of the drainage vein, which lasts until the late venous phase [7]. CECT and contrast-enhanced MRI are useful screening examinations for AVM. If the presence of AVM is suspected in these examinations, it is necessary to perform digital subtraction angiography for the definitive diagnosis of AVM [8]. Endoscopy is not useful as long as the lesions do not appear on the mucosal surface, while the color Doppler method of transrectal endoscopic ultrasonography is useful for a minimally invasive diagnosis. Asymptomatic, mild, or nonprogressive pelvic AVM can be followed and transrectal endoscopic ultrasonography is useful for monitoring [9]. There are two treatment methods: one is to stop the blood flow with TAE, and the other is to remove the AVM by surgery [10]. TAE is useful for controlling the symptoms and it is less invasive. Conventionally, cyanoacrylate adhesives such as NBCA (Nbutyl cyanoacrylate) or Onyx (ethylene vinyl alcohol polymer) have been widely used. In recent years, spherical embolic material (Embosphere ${ }^{\circledR}$ ) has been used for AVM in Japan in the wake of insurance application being adopted in $2014[6,11]$. It is a nonabsorbable embolic material, and durability of the embolic effect can be expected compared to conventionally used gelatin particles [11]. Although TAE is a palliative treatment because the nidus remains after treatment, TAE can be repeatedly performed at the time of recurrence; it is a very useful treatment alleviating symptoms and avoiding invasive surgery [10].

There is no standard surgical procedure for rectal AVM. If the lesion is in the lower rectum as in this case, APR needs to be performed as it is necessary to completely remove the AVM nidus. However, it is important to choose a surgical procedure according to the patient's condition [12]. In this case, the lesion was present in the lower rectum, so it would have been necessary to perform APR for radical cure. However, since the patient was 86 years old, we chose TAE without performing surgery. In addition to avoiding a highly invasive treatment, the improvement of the blood quality also improved the QOL of the patient.

\section{Acknowledgement}

We would like to thank Editage (www.editage.com) for English language editing.

\section{Statement of Ethics}

Not applicable. 


\section{Disclosure Statement}

We declare no conflicts of interest associated with the manuscript.

\section{Author Contributions}

Sho Ishikawa and Shoichiro Mukai wrote the manuscript; all other members contributed equally to medical treatment.

\section{References}

1 Yasui T, Oyama K, Nakano T. A case of rectal arteriovenous malformation treated successfully with transarterial embolization. Gastroenterol Endosc. 2007 Jun;49(6):1433-9.

2 Moore JD, Thompson NW, Appelman HD, Foley D. Arteriovenous malformations of the gastrointestinal tract. Arch Surg. 1976 Apr;111(4):381-9.

3 Kobayashi K, Igarashi M, Katsumata B, Yokoyama K, Sada M, Saigenji K, et al. Arteriovenous malformation of the Intestine: review of 230 cases reported in Japan. Stomach Intest. 2000 May;35(6):753-61.

4 Boley SJ, Sammartano R, Brandt LJ, Sprayregen S. Vascular ectasias of the colon. Surg Gynecol Obstet. 1979 Sep;149(3):353-9.

5 Richardson JD, Max MH, Flint LM Jr, Schweisinger W, Howard M, Aust JB. Bleeding vascular malformations of the intestine. Surgery. 1978 Sep;84(3):430-6.

6 Yamanaka N, Okamoto E, Kuwata K, Toyosaka A, Ohashi S, Hida T, et al. [A case of arterio-venous malformation of the rectum: presenting with similar findings of hemorrhoidal bleeding (author's transl)]. Nihon Shokakibyo Gakkai Zasshi. 1981 Jun;78(6):1293-7.

7 Baum S, Athanasoulis CA, Waltman AC, Galdabini J, Schapiro RH, Warshaw AL, et al. Angiodysplasia of the right colon: a cause of gastrointestinal bleeding. AJR Am J Roentgenol. 1977 Nov;129(5):789-94.

8 Parthasarathy M, Basu A, Omer AR. Massive per rectal bleeding in a child due to caecal arteriovenous malformation treated successfully by embolisation. BMJ Case Rep. 2013 Aug;2013 aug21 1:bcr2013010187.

9 Kędzierski B, Nowak G, Kuśmierska M, Jaźwiec P, Szuba A. Giant congenital malformation of the perirectal plexus in computed tomography imaging - case report. Pol J Radiol. 2013 Apr;78(2):50-3.

10 Mallios A, Laurian C, Houbballah R, Gigou F, Marteau V. Curative treatment of pelvic arteriovenous malformation - an alternative strategy: transvenous intra-operative embolisation. Eur J Vasc Endovasc Surg. 2011 Apr;41(4):548-53.

11 Saito N, Sasaki S, Shimizu T, Nozaki A, Yamatsu Y, Takahashi K, et al. Embolization with microspheres in the treatment of subcutaneous arteriovenous malformation: a case report. Sosyo. 2015 Apr;6(2):77-81.

12 Maddah G, Abdollahi A, Rouhbakhshfar 0, Taraz Jamshidi S, Hassanpour M. Arteriovenous malformations of the colon: A report of two cases and review of the literature. Caspian J Intern Med. 2017;8(1):52-5. 


\section{Case Reports in Gastroenterology}
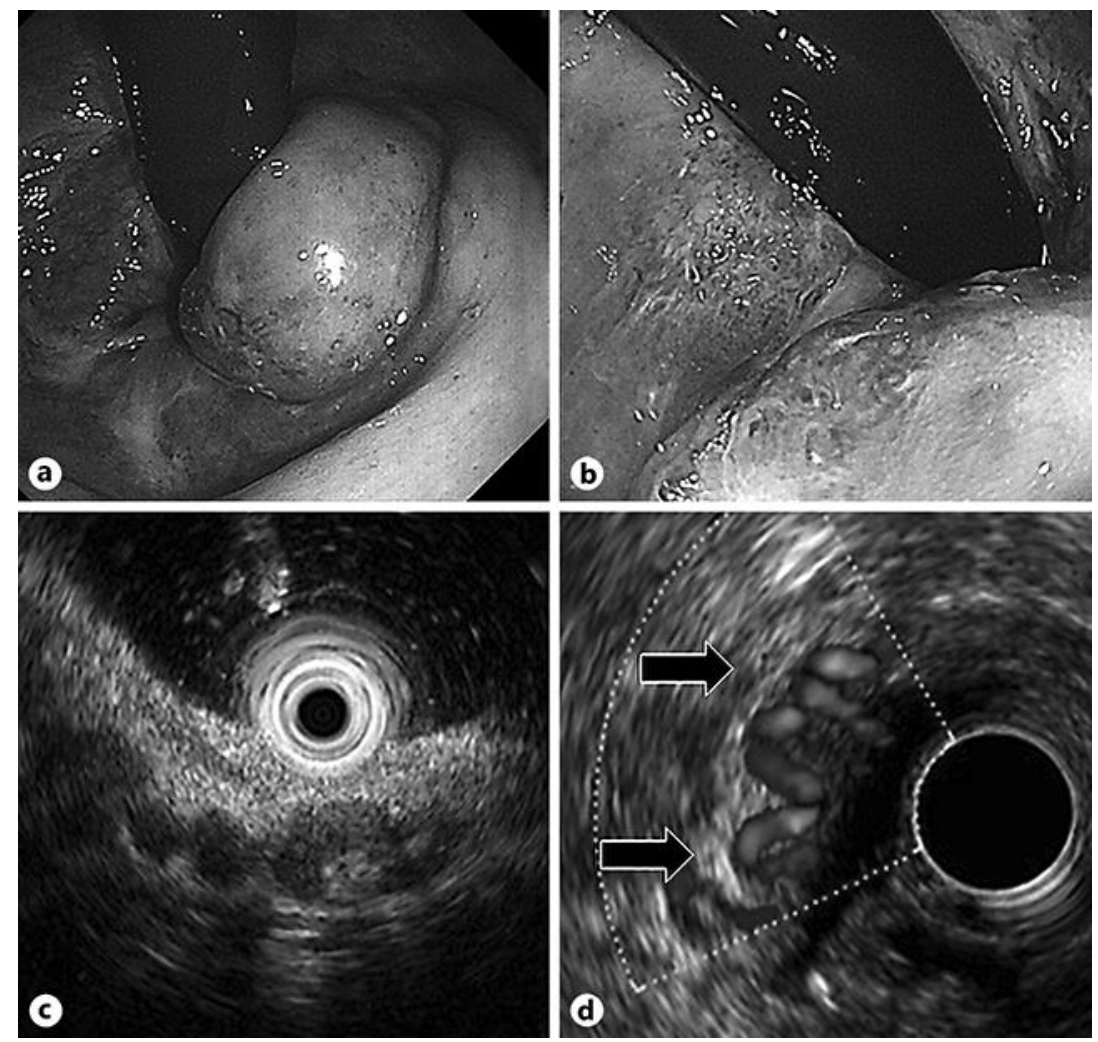

Fig. 1. a Colonoscopy revealed a submucosal tumor-like lesion in the lower rectum. $\mathbf{b}$ In the mucous membrane covering the tumor-like surface, meandering blood vessels were exposed. c Endoscopic ultrasonography revealed the lesion located outside of the rectum wall. d Color Doppler ultrasonography showed flow regions and it was considered to be a lesion rich in blood vessels. 


\section{Case Reports in Gastroenterology
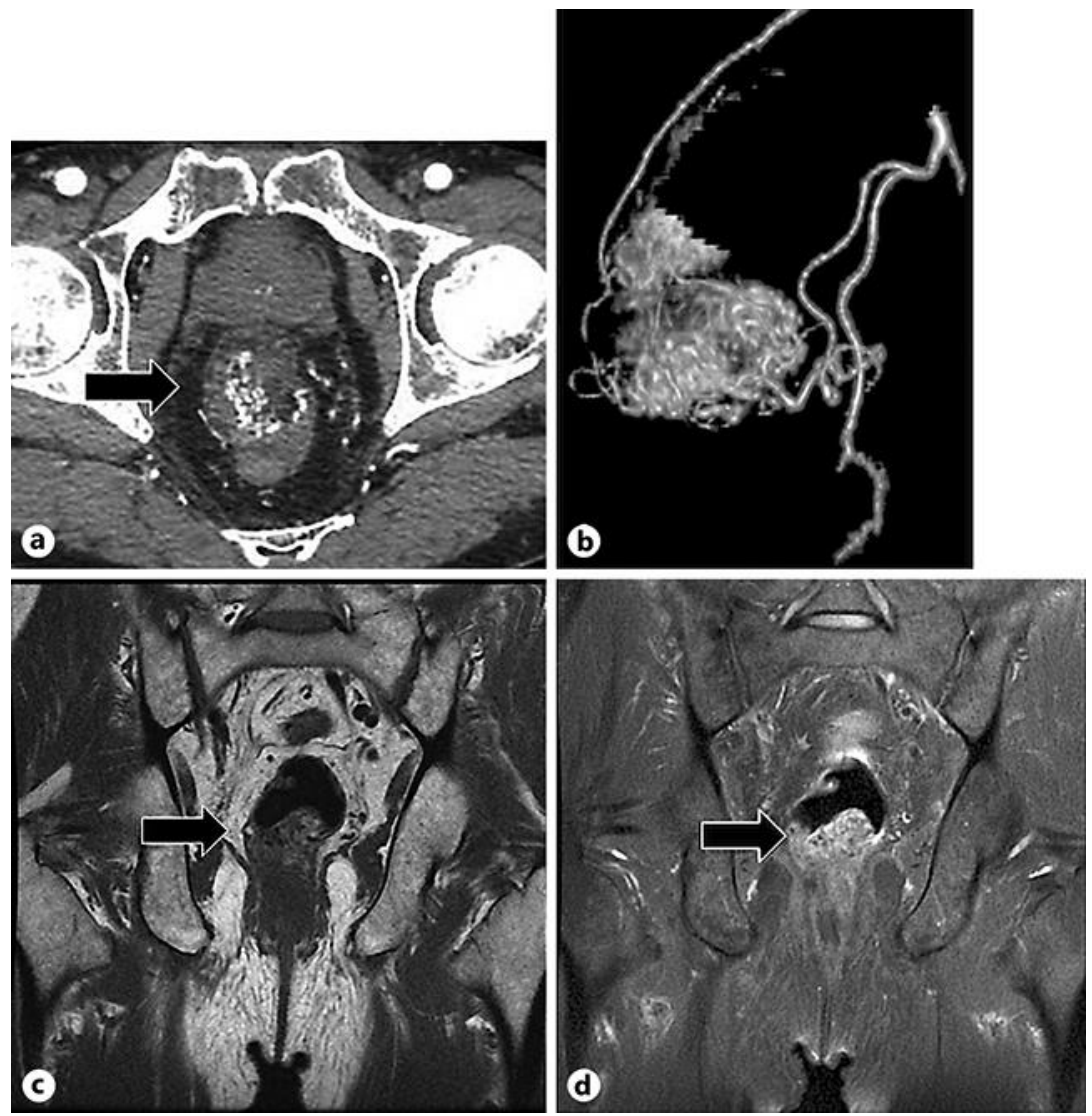

Fig. 2. a CECT revealed accumulation of abnormal vessels in the lower rectum. $\mathbf{b}$ 3D-reconstruction of the CECT showed that blood flow to the lesion was supplied from the superior rectal artery and the left middle or inferior rectal artery. c, d MRI showed low signal intensity in the thickened rectal wall on T1- and T2weighted images (T1WI and T2WI), due to which edema of the rectal wall was suspected. T1WI (c) and T2WI (d) showed flow voids in the thickened rectal wall (arrow) suggesting vascular lesion. 


\section{Case Reports in \\ Gastroenterology}

\begin{tabular}{l|l}
\hline Case Rep Gastroenterol 2020;14:7-14 \\
\hline DOI: 10.1159/000505090 & $\begin{array}{l}\text { (c) 2020 The Author(s). Published by S. Karger AG, Basel } \\
\text { www.karger.com/crg }\end{array}$ \\
\hline
\end{tabular}

Ishikawa et al.: Rectal Arteriovenous Malformation Treated by TAE
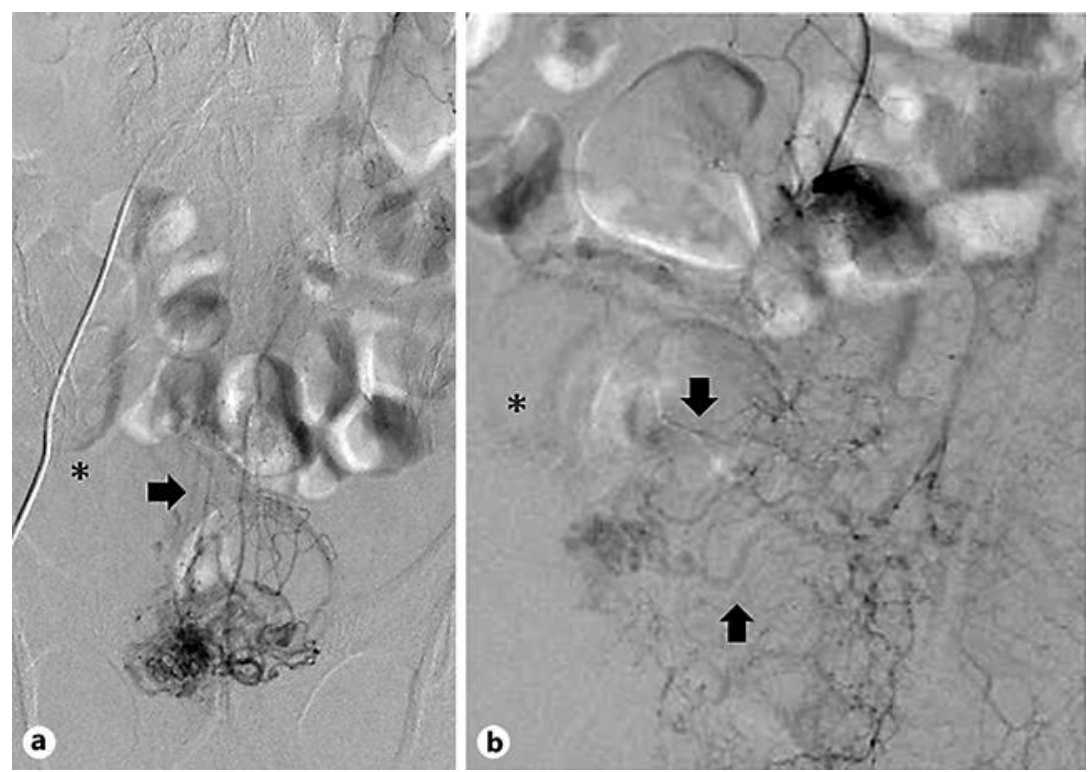

Fig. 3. a Angiography in the SRA. b Angiography in the left IIA. Pelvic angiography image showing nidus of the rectal AVM. The nidus (asterisk) was visualized by imaging of the SRA (arrow, a) and left MRA (arrow, b, upper) and IRA (arrow, b, under). 


\section{Case Reports in Gastroenterology}

\begin{tabular}{l|l}
\hline Case Rep Gastroenterol 2020;14:7-14 \\
\hline DOI: 10.1159/000505090 & $\begin{array}{l}\text { @ 2020 The Author(s). Published by S. Karger AG, Basel } \\
\text { www.karger.com/crg }\end{array}$ \\
\hline
\end{tabular}

Ishikawa et al.: Rectal Arteriovenous Malformation Treated by TAE
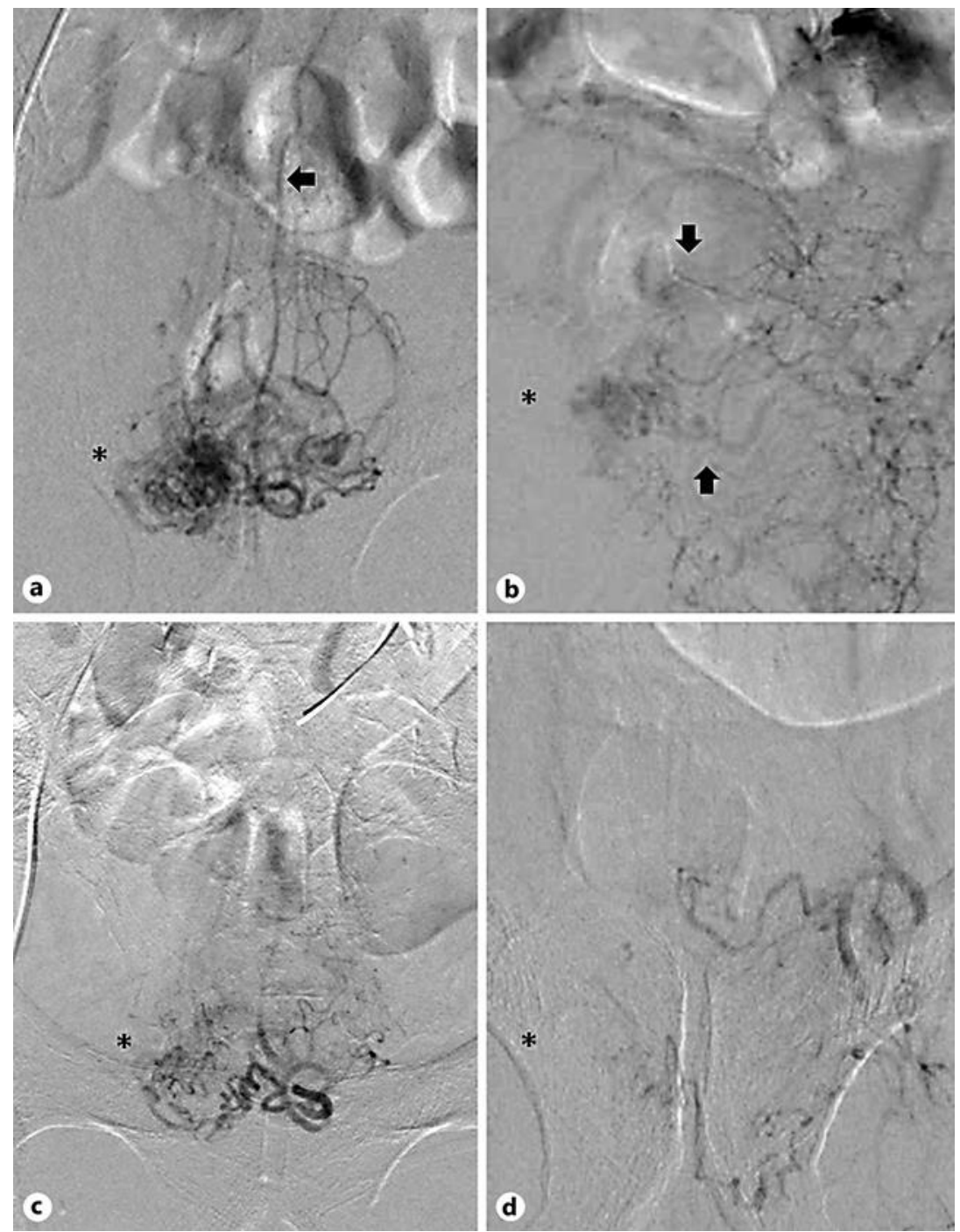

Fig. 4. a Angiography in SRA before embolization. b Angiography in SRA after embolization. c Angiography in the left IIA before embolization. $\mathbf{d}$ Angiography in the left IIA after embolization. After embolization from the SRA, left MRA and IRA, angiography showed obliteration of the blood flow into the nidus (asterisk). 